\title{
Anterior spinal hernia: an increasingly recognised cause of thoracic cord dysfunction
}

\author{
Barrie D White, John L Firth
}

Department of Neurosurgery,

University Hospital, Queen's Medical Centre, Nottingham, UK

B D White

J L Firth

Correspondence to:

Dr Barrie D White,

Department of

Neurosurgery, University

Hourpital, Queen's Medical

Centre, Nottingham

NG7 2UH, UK

Received 7 December 1993

and in revised form

7 April 1994.

Accepted 3 May 1994

\begin{abstract}
Two cases of anterior spinal hernia are presented. The medical literature is reviewed, the syndrome characterised, and its cause and treatment discussed. The patient is typically middle aged with a history of stepwise slowly progressive mid-thoracic anterior hemicord syndrome manifesting as hemianalgesia below the affected segment, followed by contralateral lower limb spasticity that develops into an asymmetric paraparesis with sparing of dorsal column sensation. Radiological investigation demonstrates an enlarged dorsal arachnoid space in association with an apparently focally narrowed thoracic cord, kinked towards the anterior dura. At operation the cord
\end{abstract}

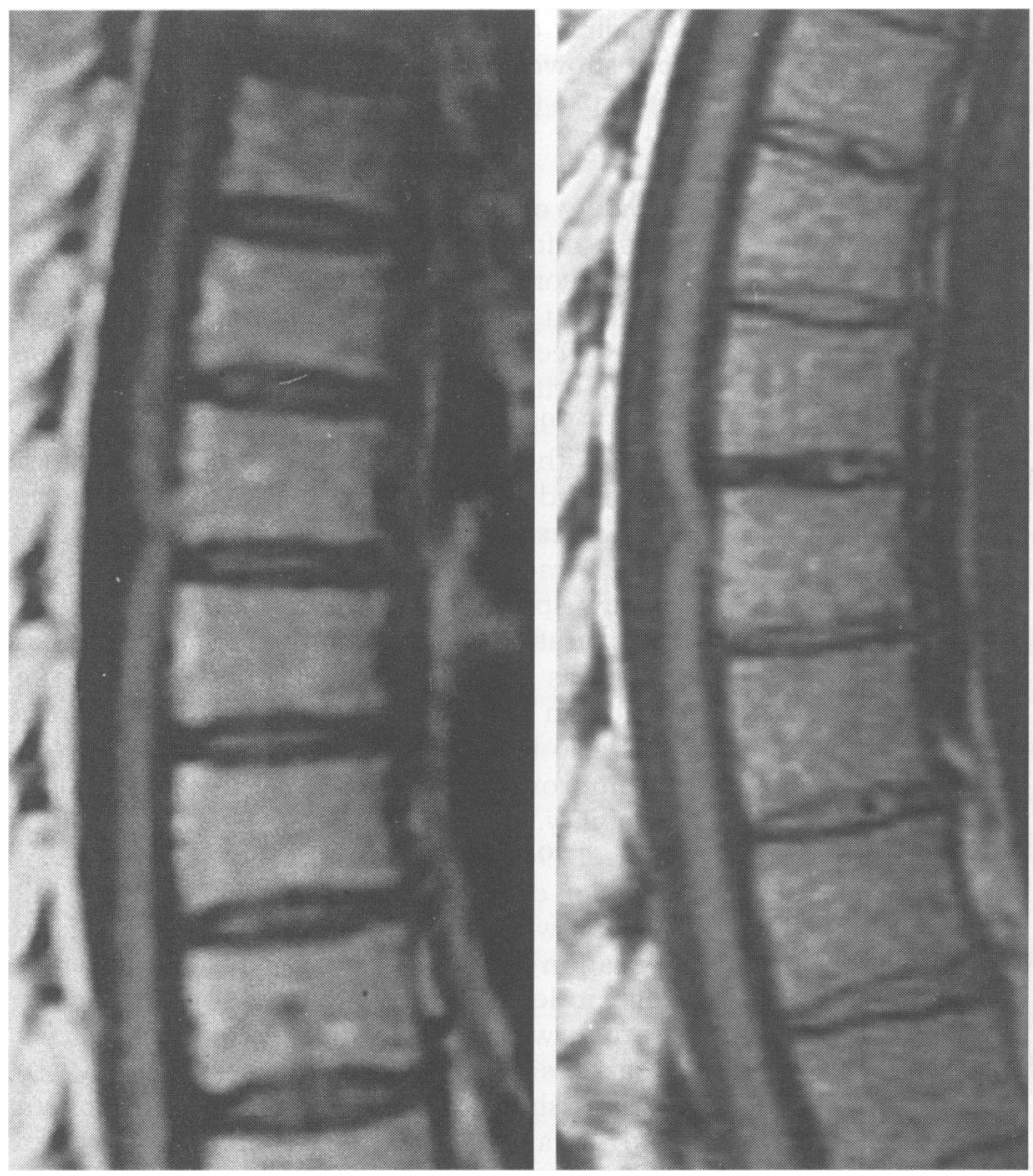

Figure 1 Left; patient 1, preoperative T1 weighted spin echo sagittal MRI showing focal anterior cord tethering at D4. Right; patient 2, preoperative T1 weighted spin echo sagittal $M R I$ showing focal anterior cord tethering at D8. is found to be prolapsed into an anterolateral dural diverticulum. The most likely cause of this syndrome is anterior spinal artery segmental branch ischaemia, in a cord chronically incarcerated in a congenital anterior meningocele. This readily treatable condition should be considered in all cases of thoracic cord dysfunction and surgical repair effected early to prevent stepwise progression to paraplegia.

$(\Im$ Neurol Neurosurg Psychiatry 1994;57:1433-1435)

Thoracic spinal cord hernia is a rare condition presenting in middle age with a typical history of anterior hemicord dysfunction preceding progressive paraparesis with preservation of posterior column sensation. The radiological findings, although subtle, are typical and surgical repair is easily effected. This condition is probably more common than currently appreciated and should be considered in all patients presenting with progressive thoracic cord dysfunction.

\section{Case reports}

PATIENT 1

In 1987 a 61 year old woman, suddenly developed a "tender warm tingling" sensation in the lateral aspect of her left leg. In 1988, after a fall caused by her legs transiently "giving way", she was found to have reduced pain and temperature sensation on the left with an upper level of T6, but otherwise normal neurology. During the next six months, she noticed her right leg becoming stiff; with impairment of walking to the point that a single stick was needed. Neurological follow up over the same period confirmed increasing lower limb spasticity, then motor deterioration to MRC4 in the right leg without tactile or proprioceptive impairment.

Magnetic resonance imaging showed the spinal cord to be acutely kinked and closely applied to the D4 vertebral body in association with dorsally placed arachnoid diverticulae (fig 1 left).

At operation, the arachnoid appeared thickened in the region of the abnormality and on filling with CSF during expiration the subarachnoid space was seen to contain multilocular dorsal arachnoid diverticulae. On 

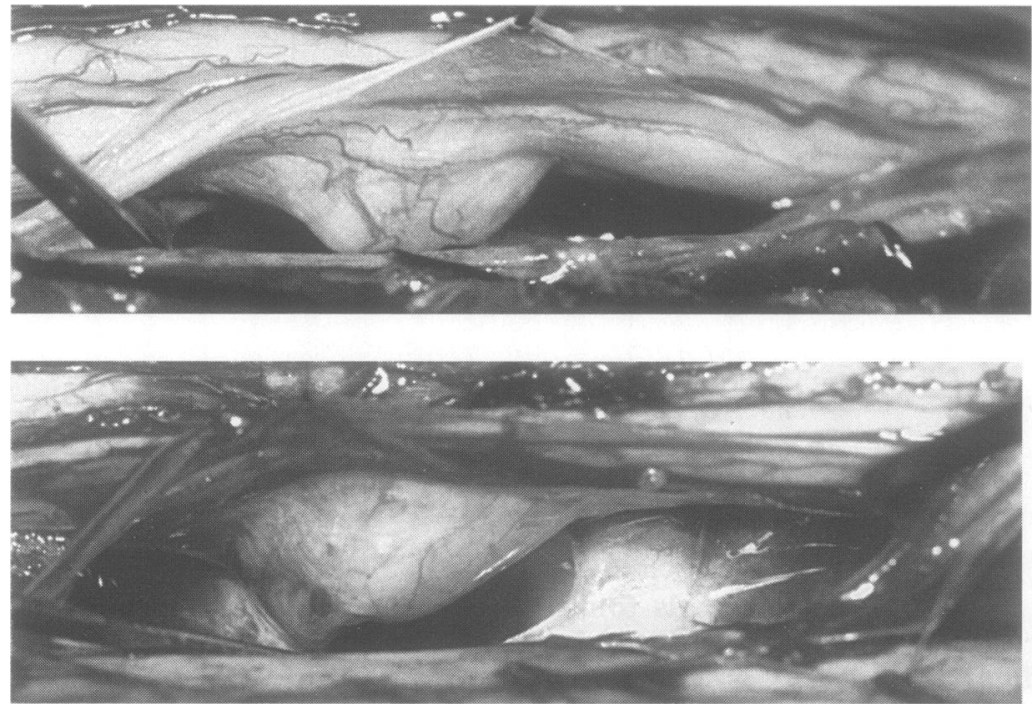

Figure 2 Top; patient 1, photograph at operation showing cord hernia delivered from anterior sharp edged oval dural diverticulum. Edge just visible to left of hernia. Bottom; patient 2, operative photograph at operation showing cord hernia delivered from anterior sharp edged oval dural diverticulum. Edge just visible to right of hermia.

opening the arachnoid the anterior cord was found to have prolapsed into, and be deformed and tightly held by a sharp edged, wide necked oval dural diverticulum slightly to the right of midline. The cord was gently delivered (fig 2 top) and to prevent recurrent prolapse a sling of fascia taken from the trapezius was passed anterior to the cord and fixed with interrupted sutures to obliterate
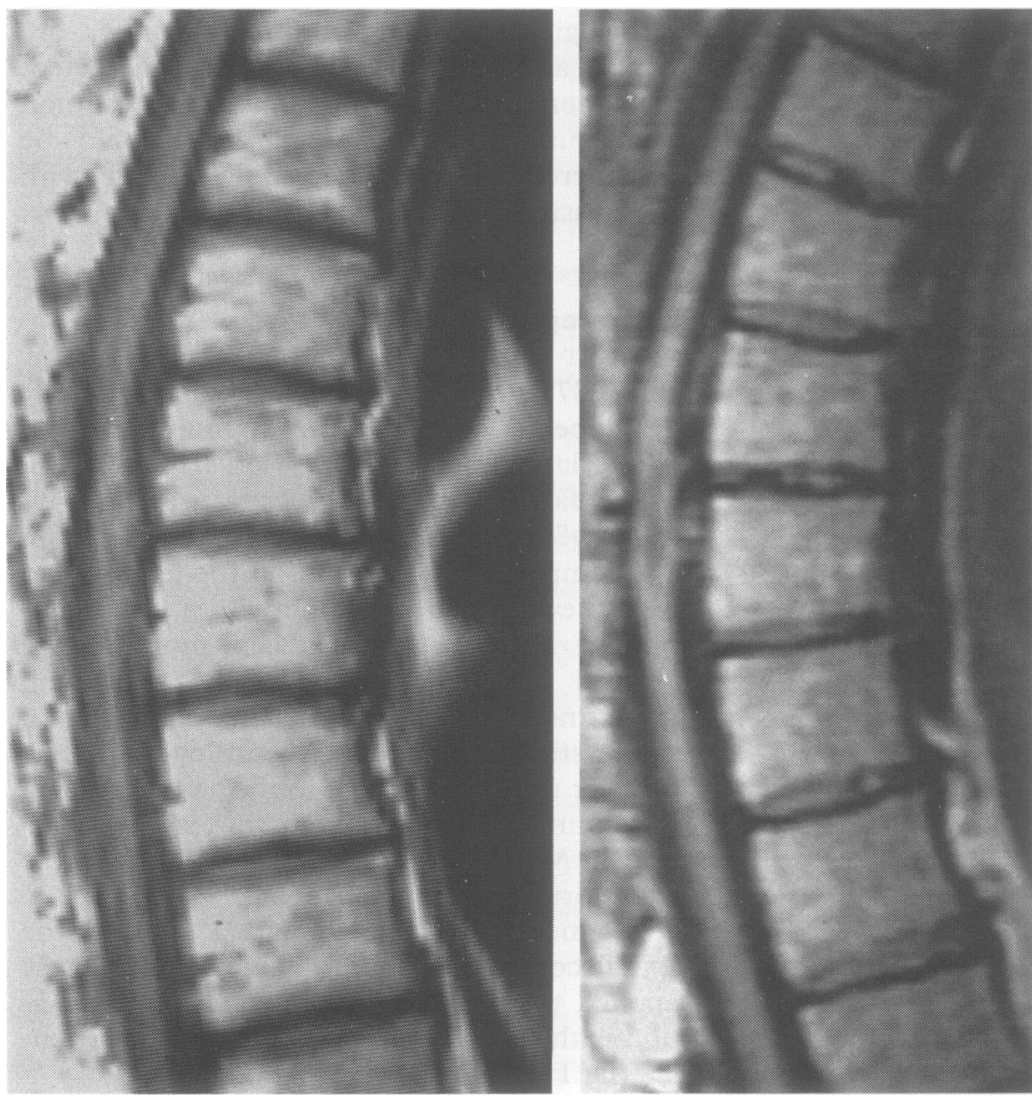

Figure 3 Left; patient 1, postoperative T1 weighted spin echo sagittal MRI showing cord realignment but persistent focal cord abnormality. Right; patient 2 , postoperative $T 1$ weighted spin echo sagittal MRI showing cord realignment but persistant focal cord abnormality. the dural opening while simultaneously acting as a hammock for the abnormal cord.

Postoperatively, the patient remains unchanged, analgesic below T6 on the left with an asymmetric spastic paraparesis affecting the right leg more than the left, but with proprioception preserved. Magnetic resonance imaging confirmed that the cord had been realigned although it remained focally abnormal (fig 3 left).

PATIENT 2

A thirty nine year old man presented in 1991, having noticed an inability to appreciate with his left leg the heat of a radiator against which he was leaning. Neurological assessment confirmed an isolated left sided spinothalamic loss with a level at T10. Stepwise progression occurred over a period of 18 months to a stiff clumsy right leg that prevented him running. Clinically during this time he developed a right anterior hemicord syndrome with preserved proprioception despite spinothalamic loss on the left below T10, right lower limb hypertonia and hyper-reflexia, ankle clonus, and an upgoing plantar (but no motor weakness).

Examination by MRI showed the abruptly kinked cord distorted towards the right anterior border of the thoracic canal at D8 and an enlarged dorsal arachnoid space (fig 1 right). Exploration confirmed the nature of the abnormality and allowed delivery and repair of the cord hernia as before (fig 2 bottom). Postoperatively there has been no change in neurological status. Magnetic resonance imaging confirmed realignment of the cord but showed a syrinx at the level of the original prolapse (fig 3 right).

\section{Review of the medical literature}

Four additional cases of anterior thoracic spinal cord hernia have been published since 1990 but the clinical syndrome has not previously been characterised or the cause of the lesion critically analysed.

In 1990, Oe et al presented a 61 year old man with a 10 year history of progressive T5 hemicord syndrome leading to an asymmetric paraparesis. ${ }^{1}$ Full investigation showed that the thoracic cord was kinked anteriorly and closely applied to the vertebral body of D4. A preoperative diagnosis of dorsal arachnoid cyst was substantiated at operation, during which an anterior dural cleft with cord hernia was also found. Both were repaired but with no improvement in the patient's longstanding disability.

In 1991, Isu et al published two cases of anterior cord hernia. ${ }^{2}$ One was a 43 year old woman with a one year history of altered pain sensation below T5 on the right and a hyperreflexic left leg. The other was a 45 year old woman with a 20 month history of right leg weakness progressing to paraparesis. Investigation showed anterior cord displacement with enlarged dorsal subarachnoid spaces in both cases.

Operation confirmed the presence of 
dorsally situated arachnoid cysts and ventrolateral dural defects with cord herniation. In both cases, surgery was confined to removal of the dorsal arachnoid cysts without dural repair. The authors postulate that the ventral dural dehiscence was the result of pressure transmitted through the cord from the dorsal intradural arachnoid cyst.

Later the same year, Tronnier et al presented a 45 year old woman with a four year history of right sided loss of pain and temperature sensation below T5. ${ }^{3}$ Magnetic resonance imaging showed the cord narrowed and closely applied to the vertebral body at D3/4, interpreted as a "...dorsally located cystic lesion displacing the spinal cord anteriorly...." At operation a left anterolateral dural dehiscence with incarcerated cord hernia was found. The hernia was reduced and the defect repaired with a dural cuff held by fibrin glue. The authors attributed the dural split to forgotten trauma.

\section{Discussion}

Six cases of this syndrome now exist in the medical literature, each with identical clinical, radiological, and surgical features. The aetiology of the dural lesion remains in doubt with previous authors either offering no explanation or invoking forgotten trauma or pressure erosion due to dorsal intradural arachnoid cysts.

Dural defects may follow trauma, but the likelihood of this passing unnoticed in the thoracic region is small because of the severity of injury required and almost invariable occurrence of neurological damage.

Similarly, it seems inconceivable that pressure sufficient to erode the dura would not, if plausible, be more commonly associated with solid intradural tumours. Furthermore, unlike extramedullary cord compression, which causes motor then global sensory change, the presenting syndrome in each of these cases relates specifically to the contents of the hernia with (at least in the initial stages) disturbed contralateral spinothalamic sensation, ipsilateral motor loss, and preserved dorsal column sensation.

The association of anterior hernia with dorsal arachnoid thickening requires explanation, but it would seem more reasonable to suggest that the arachnoid changes may be secondary to inflammation resulting from incarceration of the cord.

On the evidence available, it seems most likely that this condition is a consequence of cord prolapse into a congenital dural defect. The absolute rarity of true anterior spina bifida, the absence of somitic vertebral abnormalities in these cases, and the clinical presentation of cord herniation in middle age, however, argues against even this as a cause.

One possibility for the late presentation of this syndrome, its stepwise progression, and failure to improve after cord reduction is that the hernia is longstanding but only becomes symptomatic as a result of superadded ischaemic events affecting small distorted seg- mental branches of the anterior spinal artery in middle age.

In support of a congenital basis for this lesion is the original description by Wortzman et al, who in 1974 described a similar but more severe case involving thoracic cord prolapse into a corticated cavity likely to be a neurenteric canal remnant within the D7 vertebral body. ${ }^{4}$

The only other report cited is that of Matsuzawa et al. ${ }^{5}$ This case differs from the others in its clinical presentation, radiology, and surgical findings and seems to be a unique report of a distinct clinical entity of spontaneous cord herniation into a conventionally sited extradural arachnoid diverticulum.

\section{Conclusions}

Although the pathogenesis of the dural lesion has yet to be settled, the syndrome, which differs subtly from other causes of thoracic cord dysfunction, is well characterised by these cases.

Affecting both men and women in their middle years, it commences as a unilateral spinothalamic tract disturbance with an upper level between T4 and T10, and progresses slowly but stepwise over many months to an asymmetric paraparesis with preserved dorsal column sensation.

Imaging shows a narrowed, acutely kinked cord, closely applied to the vertebral body, in association with dorsal arachnoid abnormalities.

Surgery to deliver the cord and repair the dural defect results in stabilisation of an otherwise stepwise progressive cord vascular syndrome and should be performed as soon as the condition is suspected despite the relatively innocuous early symptoms and signs.

Of major importance is the appreciation that low pressure dorsal arachnoid abnormalities may be a marker of, or associated with ventral spinal herniation and to differentiate this clinically, radiographically, and surgically from a truly symptomatic high pressure arachnoid cyst. Failure to do so may result in inadequate surgical treatment and persistence of the (quite literally) underlying causative lesion.

Our sincere thanks are extended to Dr Alan $M$ Whitely, Dr David Jefferson (consultant neurologists) Dr Ian Holland, Dr Tim Jaspan, and Dr Geoff Narborough (consultant neuroradiologists) for their invaluable help with the care, diagnosis, and rehabilitation of these patients.

1 Oe T, Hoshino Y, Kurokawa T. A case of idiopathic herniation of the spinal cord associated with duplicated dura mater and with an arachnoid cyst. $\mathcal{F}$ fpn Orthop Assoc 1990;64:43-9.

2 Isu T, Iizuka T, Iwasaki $Y$, Nagashima $M$, Akino $M$, Abe $H$. Spinal cord herniation associated with an intradural spinal arachnoid cyst diagnosed by magnetic resonance imaging. Neurosurgery 1991;29:137-9.

3 Tronnier VM, Steinmetz A, Albert FK, Scharf J, Kunze S. Hernia of the spinal cord: case report and review of the literature. Neurosurgery 1991;29:916-9.

4 Wortzman G, Tasker RR, Rewcastle B, Richardson JC Pearson FG. Spontaneous incarcerated herniation of the spinal cord into a vertebral body: a unique cause of paraplegia. $\mathcal{F}$ Neurosurg 1974;41:631-5.

5 Masuzawa. cord herniation into a congenital extradural arachnoid cord herniation into a congenital extradural arachnoid cyst causing 